\title{
Mola Hidatiforme Completa e Eclâmpsia: Relato de Caso
}

\author{
Complete Hydatidiform Mole and Eclampsia: A Case Report \\ Izildinha Maestá ${ }^{1}$, José Carlos Peraçoli ${ }^{1}$, José Raimundo Passos ${ }^{2}$, Vera Therezinha Medeiros Borges ${ }^{1}$, \\ Carolina Diaz Pedrazzani ${ }^{1}$, Marilza Vieira Cunha Rudge ${ }^{1}$
}

\begin{abstract}
RESUM0
O desenvolvimento de pré-eclâmpsia ou eclâmpsia antes da $20^{a}$ semana deve levar à suspeita de mola hidatiforme. Descrevemos um caso de mola hidatiforme completa (MHC) e eclâmpsia concomitante em paciente com 20 anos que apresentava sangramento genital, anemia, tamanho uterino excessivo e cistos de ovário, associados a hipertensão arterial e proteinúria. Os níveis de $\beta$-hCG estavam elevados e a função tiroidiana, alterada. A ultra-sonografia mostrou-se compatível com MHC. Após o esvaziamento uterino apresentou cefaléia e alterações visuais, seguidas por convulsões tônico-clônicas que cessaram com sulfato de magnésio heptahidratado a 50\%. No seguimento pós-molar foi diagnosticado tumor trofoblástico gestacional (TTG) prontamente tratado com quimioterapia. A associação de MHC e eclâmpsia determina esvaziamento uterino imediato e seguimento pós-molar rigoroso, pelo risco aumentado de desenvolvimento de TTG.
\end{abstract}

PALAVRAS-CHAVE: Mola hidatiforme completa. Eclâmpsia. Tumor trofoblástico gestacional.

\section{Introdução}

A ocorrência de pré-eclâmpsia em gravidez precoce deve levar à suspeita de mola hidatiforme completa (MHC) ou parcial e, mais raramente, triploidia de origem não molar ${ }^{1}$. Em casos de MHC, a ultra-sonografia, associada à valores elevados da gonadotrofina coriônica ( $\beta$-hCG total), faz o diagnóstico pré-esvaziamento em $84 \%$ das vezes ${ }^{2}$. No entanto, quando se trata de pré-eclâmpsia no segundo trimestre de gestação com presença de feto malformado e placenta espessada, com ou sem áreas císticas, o cariótipo do feto é necessário para esclarecer o diagnóstico e a conduta obstétrica ${ }^{1}$.

A mola hidatiforme ( $\mathrm{MH})$ ocorre em uma de cada 1000 gestações e, na última década, a associação com pré-eclâmpsia tornou-se fenômeno raro em decorrência da rotina de exame ultra-sonográ-

${ }^{1}$ Departamento de Ginecologia e Obstetrícia - Faculdade de Medicina de Botucatu - UNESP

${ }^{2}$ Departamento de Bioestatística - Instituto de Biociências de Botucatu - UNESP

Correspondência:

Izildinha Maestá

Departamento de Ginecologia e Obstetrícia

Faculdade de Medicina de Botucatu - UNESP

Caixa Postal 530

18618-970 - Botucatu - SP fico no primeiro trimestre de gestação, que possibilita diagnóstico precoce, em fase assintomática da doença molar ${ }^{3}$.

A pesquisa bibliográfica de mais de 10 anos revelou quatro casos, sendo dois de $\mathrm{MHC}^{4,5}$ e dois de $\mathrm{MH}$ parcial (MHP) ${ }^{6,7}$. Todas as quatro pacientes tinham menos de 25 anos (entre 16 e 24 anos), eram primigestas e a idade gestacional no momento do diagnóstico foi, em média, de 16 semanas (entre 14 e 17 semanas). O tamanho do útero maior que a idade gestacional ocorreu em duas pacientes com MHC e eclâmpsia ${ }^{4,5}$. Nos dois casos de MHP e eclâmpsia, o tamanho uterino correspondeu à idade da gravide $z^{6,7}$.

$\mathrm{Na}$ avaliação do quadro clínico, alguns sinais e sintomas foram comuns, como hipertensão, cefaléia intensa, proteinúria e convulsão tônicoclônica generalizada, variando de um a três episódios convulsivos, antecedendo ${ }^{6,7}$ ou seguindo ${ }^{4,5}$ o esvaziamento uterino. O sangramento genital, as alterações visuais, a obnubilação, a dificuldade respiratória, o edema e a hiperreflexia foram observados em alguns casos. O diagnóstico de três casos foi confirmado antes do término da gravidez, pela ultra-sonografia associada à dosagem quantitativa do hCG $^{5-7}$. Em apenas um caso, o diagnóstico foi feito no momento da curetagem uterina, 
pela eliminação de vesículas ${ }^{4}$. A forma de terminação da gravidez foi curetagem uterina em três pacientes (duas MHC e uma MHP) ${ }^{4,5,7}$. A indução com prostaglandina até a expulsão do feto, seguida de extração manual da placenta, foi realizada em apenas um caso de $\mathrm{MHP}^{6}$. Vale considerar que, a recuperação do estado geral foi rápida, poucos dias após o esvaziamento uterino, em todas as pacientes com MH e eclâmpsia associada, e não ocorreu nenhuma morte materna. Três destas pacientes foram tratadas, com sucesso, com sulfato de magnésio e anti-hipertensivos ${ }^{4,5,7}$, sendo que apenas uma paciente recebeu benzodiazepínico e fenitoína para controle dos episódios convulsivos ${ }^{6}$.

O seguimento pós-molar destas pacientes, que tiveram $\mathrm{MH}$ e associação de eclâmpsia, revelou rápida normalização dos valores séricos da gonadotrofina coriônica (em torno de seis semanas pós-esvaziamento uterino) nos casos de $\mathrm{MHP}^{6,7}$. No entanto, dentre duas pacientes que tiveram MHC e eclâmpsia, em uma houve normalização dos níveis de hCG urinário em 12 semanas após o esvaziamento molar ${ }^{5}$ e outra desenvolveu tumor trofoblástico gestacional (TTG) não metastático ${ }^{4}$. Neste caso, o diagnóstico de seqüela maligna foi feito oito semanas após o diagnóstico da mola, pelo padrão da curva do $\beta$-hCG (platô em torno de $404 \mathrm{mUI} / \mathrm{mL}$ ). O tratamento foi realizado com cinco ciclos de quimioterapia (ciclos de metotrexato alternados com actinomina D), alcançando-se a remissão completa da doença ${ }^{4}$.

É alta a incidência de malignização em casos de MHC e eclâmpsia concomitante ${ }^{4,8}$. Embora se considere a eclâmpsia como fator de risco independente para TTG ${ }^{8}$, observa-se associação de outros fatores em casos de MHC e eclâmpsia: idade gestacional média de 16 semanas, altura uterina igual ou superior a 20 centímetros, tamanho uterino maior que o esperado para a idade gestacional, cistos de ovário, e níveis elevados de $\beta-\mathrm{hCG}^{4}$. Segundo Newman e Eddy ${ }^{8}$, dentre 58 casos de MHC complicados com eclâmpsia, $8(13,8 \%)$ evoluíram para óbito, $30(51,7 \%)$ não tiveram relato de seguimento pós-molar adequado e 20 (34,5\%) foram acompanhadas e, destas, 16 (80\%) tiveram TTG.

Considerando-se a raridade da associação de MHC e eclâmpsia, este trabalho relata e discute um caso tratado e acompanhado no Hospital das Clínicas da Faculdade de Medicina de Botucatu (HC-FMB).

\section{Relato do Caso}

Paciente com 20 anos, sem antecedentes mórbidos; secundigesta, primípara; na $15^{\mathrm{a}}$ semana de gestação, apresentava no acompanhamen- to pré-natal de baixo risco, realizado em Unidade Básica de Saúde, crescimento uterino exagerado e ausculta fetal negativa. A ultra-sonografia mostrou massa heterogênea intra-uterina, com estruturas anecóicas arredondadas e ecos amorfos, compatíveis com MHC.

Encaminhada para tratamento no Hospital das Clínicas da Faculdade de Medicina de Botucatu, foram verificados hipertensão arterial (160/110 mmHg), edema periférico, palidez cutâneo-mucosa, altura uterina de $26 \mathrm{~cm}$ (desproporcional para a idade gestacional), sangramento genital escasso e cistos de ovário bilaterais. Novo exame ultra-sonográfico confirmou o aspecto de vesículas, com dopplervelocimetria revelando baixa resistência ao fluxo nas artérias uterinas.

Os exames complementares, na admissão da paciente, tiveram como resultado: tipagem sangüínea - O Rh positivo; hematócrito - 25,8\%; hemoglobina - 8,7 g\%; proteinúria - 2,39 g/24 h; ácido úrico - 7,5 mg/dL; $\beta$-hCG total - $408.208 \mathrm{mUI} /$ mL; TSH - 0,05 mUI/mL; T4 livre - 3,63 ng/dL. A radiografia de tórax e a tomografia computadorizada do crânio não revelaram alterações. A fundoscopia mostrou edema retiniano.

Feito o diagnóstico de MHC, foi realizado esvaziamento uterino por vácuo-aspiração. O material aspirado pesou 1.450 g e mediu, em conjunto, $30 \times 20 \times 5 \mathrm{~cm}$, consistindo de estruturas vesiculares com diâmetros variados (até $2 \mathrm{~cm}$ ) preenchidas por líquido seroso, claro, permeadas por coágulos sangüineos. No exame histopatológico do tecido molar foram preenchidos os critérios clássicos de Szulman e Surti $(1978)^{9}$ : ausência de embrião e âmnio, presença de hiperplasia trofoblástica difusa e vilos hidrópicos e avasculares com cavitações císticas centrais.

No pós-operatório imediato (12 h após o esvaziamento uterino) apresentou cefaléia e alterações visuais seguidas por convulsões tônicoclônicas (três episódios), que cessaram com administração de sulfato de magnésio heptahidratado a 50\% (dose de ataque de $4 \mathrm{~g}$ intravenoso e manutenção com $2 \mathrm{~g}$ de $2 / 2 \mathrm{~h}$, por via intravenosa). A paciente teve melhora dos niveis pressóricos com uso do anti-hipertensivo maleato de enalapril. Recebeu alta hospitalar no $6^{\circ}$ dia de internação, com retorno semanal agendado no seguimento pós-molar e orientação para uso de contracepção hormonal de baixa dosagem.

A pressão arterial, o edema periférico e a proteinúria se normalizaram três semanas após o esvaziamento molar. Entretanto, observou-se subinvolução uterina e persistência dos cistos de ovário, apesar de estar assintomática. A curva de regressão do $\beta$-hCG mostrou queda inicial, com valor do $\beta$-hCG total de $4.797 \mathrm{mUI} / \mathrm{mL}$, se- 
guida por aumento dos valores do marcador biológico da doença na $4^{\mathrm{a}}$ e $6^{\mathrm{a}}$ semanas seguintes, 6.148 e $7.860 \mathrm{mUI} / \mathrm{mL}$, respectivamente. $\mathrm{Na} 4^{\mathrm{a}}$ semana de seguimento, a ultra-sonografia revelou útero com volume de $267 \mathrm{~cm}^{3}$, cistos de ovário bilaterais (ovário direito $-75 \mathrm{~cm}^{3}$, ovário esquerdo - $46 \mathrm{~cm}^{3}$ ) e circulação uterina com resistência diminuída (SD artéria uterina direita = 1,85; SD artéria uterina esquerda $=1,77$ ). As evoluções clínica e laboratorial, associadas à ultrasonografia, levaram ao diagnóstico de TTG. A investigação de metástases com radiografia de tórax e ultra-sonografia abdominal foi negativa. A classificação do TTG foi estádio I (FIGO) de baixo risco (OMS).

Optou-se pelo uso de quimioterapia por agente único com metotrexato com resgate de ácido folínico (MTX-FC), em regime ambulatorial. Foram realizados quatro ciclos de MTX-FC, sendo necessários dois ciclos adicionais de actinomicina-D, em decorrência de resistência verificada pela manutenção dos niveis de $\beta$-hCG total. Antes de cada ciclo de quimioterapia, foram avaliados o peso da paciente, hemograma, função renal e hepática e $\beta$-hCG total. Os efeitos colaterais consistiram de náuseas e vômitos, controlados com uso de ondasetron. A remissão da doença, verificada por três dosagens consecutivas de $\beta$-hCG total (nivel de corte de $5 \mathrm{mUI} / \mathrm{mL}$ ), foi alcançada com 28 semanas pós-esvaziamento uterino. O seguimento após a quimioterapia foi feito durante um ano, com determinação mensal do $\beta$-hCG total, exame ginecológico e contracepção. Após este período a paciente recebeu alta do acompanhamento, sendo liberada para gravidez.

\section{Discussão}

A pré-eclâmpsia/eclâmpsia é complicação grave da MHC e é causada pela acentuada hiperplasia trofoblástica, caracterizada pelo tamanho exagerado do útero volumoso, efeito da gonadotrofina sobre os ovários (cistos tecaluteínicos) e níveis séricos elevados de $\beta$-hCG to$\operatorname{tal}^{4,8}$. Relatos históricos ressaltavam incidência aumentada de pré-eclâmpsia em portadoras de $\mathrm{MH}$, com variação entre 12 e $30 \%^{10}$. Em contraste, relatos das duas últimas décadas mostram diminuição significativa nos casos de MHC complicada com pré-eclâmpsia, uma vez que nos dias de hoje o diagnóstico é feito no primeiro trimestre, em geral antes do aparecimento das manifestações sistêmicas da $\mathrm{MHC}^{3}$. Portanto, a eclâmpsia em associação com MH é evento raro. Este é o primeiro caso no HC-FMB em 10 anos de experiên- cia do centro de referência para DTG, o que significa incidência de 0,9\% entre as molas tratadas.

Curry et al. ${ }^{10}$ mostram estreita correlação entre útero volumoso, pré-eclâmpsia e TTG. MHC de alto risco para TTG é caracterizada por tamanho uterino maior que a idade gestacional, nível de gonadotrofina coriônica maior que 100.000 $\mathrm{mUI} / \mathrm{mL}$, cistos de ovário com mais de $6 \mathrm{~cm}$ de diâmetro e complicações médicas, tais como préeclâmpsia, embolização trofoblástica e hipertiroidismo. Nessas pacientes, a incidência de TTG é de 40 a $50 \%{ }^{11}$. Estudo mais recente ${ }^{12}$ evidenciou que pacientes com $\mathrm{MHC}$ de alto risco têm risco 3,5 vezes maior para desenvolver TTG, em comparação com as de baixo risco.

Quando existe MHC com eclâmpsia concomitante observa-se associação de fatores de risco para TTG e, como conseqüência, incidência elevada de malignização ${ }^{4,8}$. A paciente do presente caso apresentava útero volumoso, cistos de ovário com diâmetros maiores que $6 \mathrm{~cm}, \beta$-hCG total de 408.000 $\mathrm{mUI} / \mathrm{mL}$, hipertiroidismo e eclâmpsia. Desenvolveu TTG, diagnosticado precocemente no seguimento pós-molar pelos niveis de $\beta$-hCG em ascensão, em fase ainda assintomática. A remissão completa da doença foi alcançada com quimioterapia por agente único (metotrexato/actinomicna-D) 28 semanas pós-esvaziamento uterino. Após período de um ano com manutenção de niveis séricos normais de $\beta$-hCG total, recebeu alta, sendo liberada para gravidez. Outros casos descritos na literatura tiveram evolução semelhante ${ }^{4,8}$.

Os critérios diagnósticos utilizados para TTG pós-MHC e eclâmpsia variam nos relatos $^{8}$. No entanto, a determinação seriada da gonadotrofina coriônica é imprescindivel para o diagnóstico de malignidade em estágio precoce e assintomático ${ }^{13}$.

O controle das pacientes com $\mathrm{MH}$ e eclâmpsia concomitante deve ser com sulfato de magnésio hepta-hidatratado a 50\%, medicamento de escolha para cessar o quadro convulsivo e melhorar o prognóstico materno ${ }^{14}$. Anti-hipertensivos podem ser necessários para controle da pressão arterial. A conduta obstétrica deve ser o esvaziamento uterino imediato para interromper a evolução da pré-eclâmpsia. A vácuo-aspiração é o método de escolha pelo menor tempo cirúrgico e menor risco de complicações, como hemorragia e perfuração uterina.

Na alta hospitalar, a conscientização da paciente e de sua família é de importância para adesão ao acompanhamento proposto. Além disso, é necessária orientação de contracepção durante um ano. Vale ressaltar que, em casos de MHC com associação de eclâmpsia, o seguimento pós-molar deve ser mais rigoroso pelo maior risco de desenvolvimento de $\mathrm{TTG}^{4,8,11}$. 


\section{ABSTRACT}

Development of preeclampsia/eclampsia prior to 20 weeks of pregnancy should raise the suspicion of hydatidiform mole. We report a case of complete hydatidiform mole (CHM) concurrent with eclampsia in a 20-year-old patient with vaginal bleeding, anemia, large uterine size, and ovary cysts associated with hypertension and proteinuria. Plasmatic $\beta$ $h C G$ levels were high and there was abnormal thyroid function. The ultrasonographic findings were compatible with CHM. After uterine evacuation, the patient had headache and visual alterations, followed by tonic-clonic seizures, which ceased with the administration of $50 \%$ magnesium sulfate. At post-molar follow-up, a gestational trophoblastic tumor (GTT) was diagnosed and promptly treated with chemotherapy. Association between CHM and eclampsia requires immediate uterine evacuation and strict post-molar follow-up, due to increased risk of GTT development.

KEYWORDS: Complete hydatidiform mole. Eclampsia. Gestational trophoblastic tumor.

\section{Referências}

1. Rijhsinghani A, Yankowitz J, Strauss RA, Kuller JA, Patil S, Williamson RA. Risk of preeclampsia in second-trimester triploid pregnancies. Obstet Gynecol 1997; 90:884-8.

2. Lindholm H, Flam F. The diagnosis of molar pregnancy by sonography and gross morphology. Acta Obstet Gynecol Scand 1999; 78:6-9.

3. Coukos G, Makrigiannakis A, Chung J, Randall TC, Rubin SC, Benjamin I. Complete hydatidiform mole. A disease with a changing profile. J Reprod Med 1999; 44:698-704.
4. Newman RB, Eddy GL. Association of eclampsia and hydatidiform mole: case report and review of the literature. Obstet Gynecol Surv 1988; 43:185-90.

5. Tedesco JTA, Wajntraub I, Faria Júnior D. Mola hidatiforme completa concomitante com eclâmpsia precoce: relato de caso e revisão de literatura. J Bras Ginecol 1995; 105:351-6.

6. Slattery MA, Khong TY, Dawkins RR, Pridmore BR, Hague WM. Eclampsia in association with partial molar pregnancy and congenital abnormalities. Am J Obstet Gynecol 1993; 169:1625-7.

7. Ramsey PS, Winter JT, Gaffey TA, Ramin KD. Eclampsia complicating hydatidiform molar pregnancy with a coexisting viable fetus. A case report. J Reprod Med 1998; 43:456-8.

8. Newman RB, Eddy GL. Eclampsia as a possible risk factor for persistent trophoblastic disease. Gynecol Oncol 1989; 34:212-5.

9. Szulman AE, Surti U. The syndromes of hydatidiform mole. II. Morphologic evolution of the complete and partial mole. Am J Obstet Gynecol 1978; 132:20-7.

10.Curry SL, Hammond CB, Tyrey L, Creasman WT, Parker RT. Hydatidiform mole: diagnosis, management, and long-term follow-up of 347 patients. Obstet Gynecol 1975; 45:1-8.

11.Goldstein DP, Berkowitz RS, Bernstein MR. Management of molar pregnancy. J Reprod Med 1981; 26:208-12.

12.Maestá I, Rudge MVC, Abreu ES, Dalben I, Peraçoli JC. Preditores clínicos e histopatológicos de tumor trofoblástico gestacional pós-mola hidatiforme completa. Rev Bras Ginecol Obstet 2000; 22:167-73.

13.Maestá I, Rudge MVC, Passos JRS, Calderon IMP, Carvalho NR, Consonni M. Características das curvas de regressão da gonadotrofina coriônica pósmola hidatiforme completa. Rev Bras Ginecol Obstet 2000; 22:373-80.

14. Which anticonvulsivant for women with eclampsia? Evidence from the Collaborative Eclampsia Trial. Lancet 1995; 345:1455-63.

Recebido em: 14/11/2003 Aceito com modificações em: 25/6/2003 